\title{
The Role of Cognitive Computing and Collective Cognitive Computing in the Future Internet
}

\author{
Andy E. Williams ${ }^{1, *}$ Nenad Petrovic ${ }^{2}$ \\ ${ }^{1}$ Nobeah Foundation, Nairobi, Kenya \\ ${ }^{2}$ University of Niš, Faculty of Electronic Engineering, Niš, Serbia
}

\begin{abstract}
INTRODUCTION: The European Union, the United States, and China have all had, or currently have, programs to support research into the development of their vision of the Internet of the future.

OBJECTIVES: To explore whether and if so how cognitive computing or collective cognitive computing is likely to be a critical aspect of all of these visions of the future Internet, even where these visions differ wildly.

METHODS: An analysis is performed to assess whether and how AGI and GCI might be used to optimize achievement of the EU's vision for a future Internet. And an analysis is performed to determine whether and if so how AGI and GCI might be used to optimize achievement of China's potentially very different vision for a future Internet.

RESULTS: AGI and GCI appear to create the possibility of future Internet technology that selfassembles in different ways according to the users, so that it simultaneously might maximize outcomes in implementing potentially conflicting visions of that future Internet.

CONCLUSION: The conceptual example of customizing a news media website for two individual users of opposite political persuasions suggests that while the overhead of customizing such services might potentially result in massively increased storage and processing overhead, within a network of cooperating services in which this customization reliably creates value, this is potentially a significant opportunity.
\end{abstract}

Keywords: General Collective Intelligence, Human-Centric Functional Modelling, Artificial General Intelligence, customization, individualization, products, services

\section{Introduction}

A recently developed Human-Centric Functional Modeling (HCFM) [9] approach enables complex systems with stable functionality to be modeled in terms of a minimal set of reversible functions through which they can change their state. The accessible states form a "functional state space" through which the system moves. By defining boundary conditions on this movement, it is possible to define models representing all the behavior a complex system is capable of, without even knowing how that functionality is implemented. This HCFM approach has been used to develop what is believed to be the first model of individual human or artificial cognition with the potential capacity for human-like general problem-solving ability, as well as what is believed to be the first model of collective cognition with the potential capacity for general problem-solving ability (intelligence) that is exponentially greater than that of any individual in the group.

Functional state spaces can be created to define a set of operations capable of representing all behavior in any hardware or software application domain, for example the blockchain domain, or the communication domain. Combined with an

"Corresponding author. Email: awilliams@nobeahfoundation.org 
intelligent agent based on some subset of functionality required by an AGI, where that agent execute operations within that domain on the user's behalf in order to optimize individual user outcomes, this becomes a cognitive computing application (e.g. a cognitive communication application, or a cognitive blockchain application). Combined with a platform based on some subset of functionality required by a General Collective Intelligence or GCI, where that GCI orchestrates execution of operations within that domain in order to optimize collective outcomes (outcomes for all users), this becomes a collective cognitive computing application (e.g. a collective cognitive communication application also known as a cognitive communication platform, or a collective cognitive blockchain application also known as a cognitive blockchain platform).

AGI has the potential to exponentially increase problem-solving ability over that of any individual, but the outcomes achieved by that AGI might be aligned with the interests of a centralized entity (e.g. its owner). Because GCI has the potential to exponentially increase the group's ability to impact any problem in general, GCI is predicted to be of great importance to the future Internet and to computing in general [12]. This paper explores how cognitive computing and collective cognitive computing might be applied to developing the future Internet.

\section{Related Work}

The functional modelling approach referred to in this paper has been used to define what is believed to be the first model of Artificial General Intelligence or AGI [10] with the potential capacity for human-like general problem-solving ability, as well as a model for General Collective Intelligence or GCI with the potential for exponentially greater general problem-solving ability (intelligence) than any individual. Though others have described the concept of a general collective intelligence factor (c factor) [17], though at least one other has defined a model for a general collective intelligence factor [18], and though others have described the concept of a collective super intelligence [19], to the author's knowledge the author's own work is the only model that defines the specific mechanisms required for a GCI with the capacity to achieve super intelligence in that general collective intelligence factor. Because of this, and because this model is so new, all of the existing literature surrounding GCI refers to the author's own published work, or pre-prints of the author's unpublished work that is still under review.

\section{Research Questions and Approach}

The research questions addressed in this paper are approached with the method described below:

Section 3: Research Question Addressed

3.1 Is cognitive computing or collective cognitive computing likely to be a critical aspect of different visions of the future Internet, even where, or particularly where these visions differ wildly?

Section 4: Model

4.1. Distinguishing cognitive computing from other models.

4.2. Distinguishing collective cognitive computing from other models.

4.2. Existing approaches for modelling the Internet.

Section 5. Method

5.1. An analysis is performed to assess how functional models might be individualized.

5.2. An analysis is performed to assess whether the truth of individualization requirements might be determined.

5.3. An analysis is performed to assess whether individualization might be optimized according to those requirements.

Section 6. Findings

Section 7. Research Limitations

Section 8. Practical Implications

Section 9. Conclusions

\section{Model}

A full discussion of the functional modelling approach is too broad for a single paper. Aspects 
of the approach that are relevant to the research questions are described below.

\subsection{Distinguishing Cognitive Computing From Other Models}

From the functional modeling perspective, cognitive computing with software involves the decomposition of software functionality into a hierarchy of functional domains. Each functional domain is represented by a finite set of functions that together can represent anything that any software in that domain can do. Any software is then an implementation of these computing operations. These implementations can vary infinitely, but because they are represented by a common functional model, all implementations of such computing operations can be executed on behalf of the user by an intelligent agent implementing some subset of AGI functionality. The same holds true for hardware. Computing within a system of cognition on the other hand describes an intelligent agent executing an unbounded (and therefore unpredictable and nontransparent) set of operations. Being a black box, these operations can only be optimized by the system owner. They can't be optimized by the end user so as to maximize whatever outcomes they are targeting. In effect, the user interacts with the system owner, and not directly with the system, and might have little ability to change the interaction in a way that optimizes their individual outcomes.

\section{Cognitive Computing vs Computing in a System of Cognition}

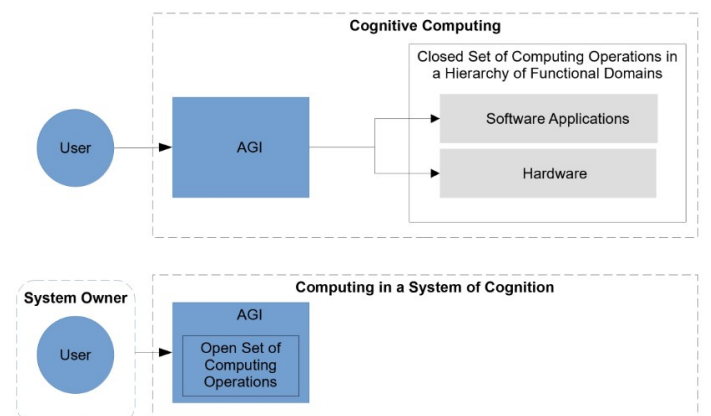

Figure 1. Cognitive computing vs computing in a centralized system of cognition..
The difference between a decentralized Internet application that maximizes the user's individual outcomes and an Internet application governed with the help of advanced AI and AGI by a single application provider as a centralized entity, depends on many factors. One might assume the individual or centralized application owning entity are benevolent, in which case the individual's rights and freedom to choose their own outcomes might not be so out of line with having a centralized entity choose those outcomes for them. Or one might assume that one or the other are malevolent. If it is the individual who is malevolent, then removing their rights and freedoms might be justified. If it is the application owning entity that is malevolent, then removing its capacity to impose itself might be justified. But rather than making an assumption that either approach is correct in all cases, a more optimal approach is to define a cognitive Internet application that simply chooses whatever implementation of any component for solving any problem in the domain of Internet application where that solution is optimal.

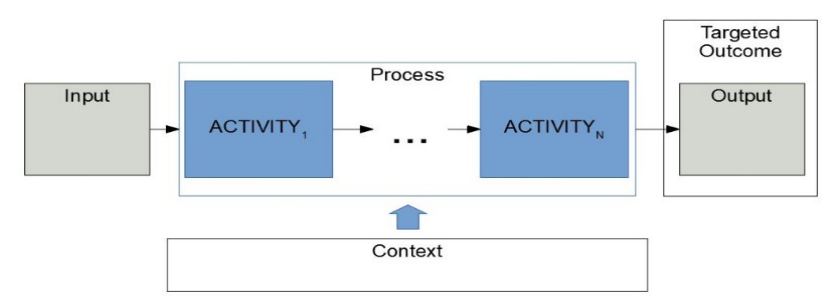

Figure 1. Functional model of the process implemented by a product or service interacting with a user.

Representing collective reasoning processes as having activities executed by individuals within roles enables the most important properties of execution to be decoupled from any particular instance of execution so that outcomes might be scaled. Aside from this very general representation, a more detailed representation of reasoning processes and of the information 
passing in and out of those processes is provided by the Functional Modelling Framework which defines the building blocks that all semantic models must be comprised of from a functional modelling of view. But consideration of this level of detail is not necessary here and is outside of the scope of discussion.

\subsection{Collective Cognitive Computing}

From the functional modeling perspective, collective cognitive computing with software involves the orchestration of collective processing by a GCI. Individuals participate directly in these collective processes, or an intelligent agent implementing some subset of AGI functionality and within the user's sole control engages in this participation on their behalf. All collective processes, including the process of constructing the GCI itself, are represented in the same common way. That is, processes are represented in terms of the same hierarchy of functional domains used in cognitive computing, and the same finite set of functions within each domain that together can represent anything that any software in that domain can do. This transparency ensures that processes can be collectively optimized.

\section{Collective Cognitive Computing}

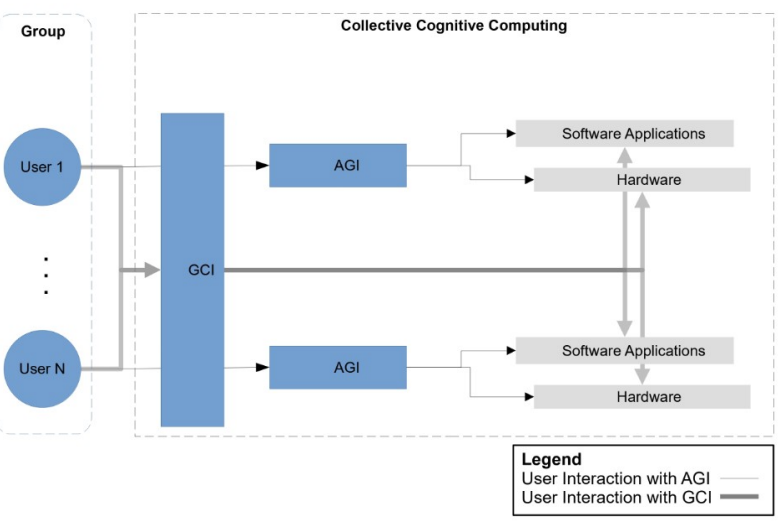

Figure 2. Collective cognitive computing provides the decoupling of all aspects of process execution that is required for collective process optimization.
Collective computing within a system of cognition on the other hand describes an intelligent agent executing an unbounded (and again unpredictable and non-transparent) set of operations on behalf of the system owner. Because the inner workings of this agent are not transparent to the group, its operations can only be optimized by the system owner, and cannot be collectively optimized. Again, in the case of a cognitive Internet platform, this might constrain Internet services to serve centralized interests.

The difference between a decentralized Internet that maximizes outcomes for all users collectively and an Internet governed by a centralized entity with the help of advanced AI and AGI, again depends on many factors. But again, because AI is a black box to users, and because black boxes can neither be optimized nor detected when their behavior is malicious or otherwise sub-optimal, rather than making an assumption that either is correct in all cases, a more optimal approach is to define a collective cognitive Internet application (e.g. a cognitive Internet platform or simply "cognitive Internet) that simply chooses whatever implementation of any solution to any problem in the Internet domain where that solution is optimal.

\section{Collective Cognitive Computing vs Collective Computing in a System of Cognition}

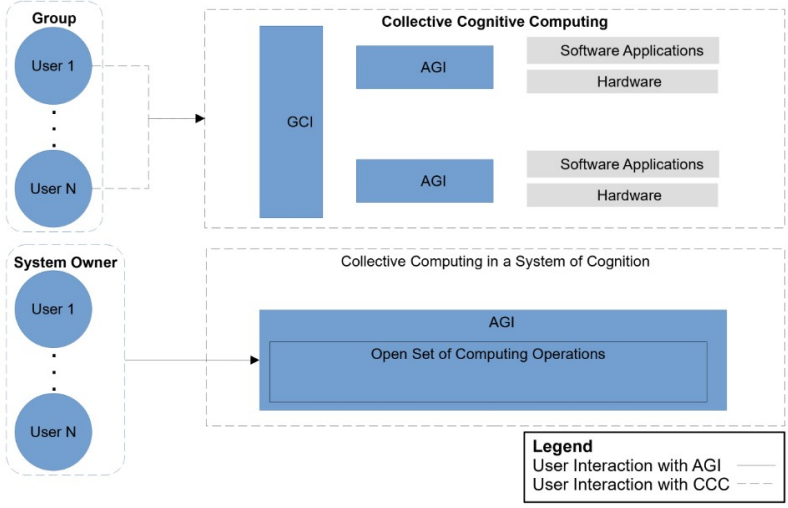

Figure 3. Whereas no entity can prevent collective optimization of processes in collective cognitive computing, in collective computing in a centralized system of cognition no individual other than the system owner can allow or achieve that collective optimization. 
Whether this difference is potentially a "point of no return" is unknown. In the case of the cognitive Internet, or in any other collective computing in a centrally owned system of cognition, no individual other than the system owner can allow or achieve collective optimization. The power of such an approach to invisibly remove even the possibility of other options might potentially make such a system effectively permanent. Or at least permanent until the entity in charge decides to change the system or is no longer present. In a system of collective cognitive computing however, no entity can prevent collective optimization of processes as required to maximize collective outcomes.

\subsection{Existing Approaches for Modelling the Internet}

A brief review of the literature was conducted to survey where Domain Specific Modelling and other modelling methods have been applied to represent aspects relevant to the Internet of future. Among them, both the commercial projects and results of individual research will be covered.

European Commission's Framework Programme for Research and Innovation known as Horizon 2020, has brought numerous projects with contributions in area of Future Internet - from theoretical models to industry-ready solutions, mainly focusing on the development and adoption of novel Fog computing paradigm. It refers to computing architectures and services which aim to leverage the computation power provided by devices residing within the Edge of the network in order to overcome network latency issues and data movement constraints due to privacy and legal regulations [21] (such as GDPR [22]), which are the main drawbacks of the existing Cloud computing model. Fog computing is recognized as one of the key-enablers of Industry 4.0 [23], smart cities [24] and smart grids [25] which are all of utmost strategic importance for EU.

Moreover, two novel networking paradigms play an important role in the development of Future Internet: Network Function Virtualization (NFV) and Software-Defined Networking (SDN) [26]. The first concept refers to the execution of functions traditionally performed by dedicated networking hardware (routers, switches, firewalls) using commodity servers, instead. On the other side, SDN is a concept of separating data from control plane, so that control plane becomes programmable enabling the embedding of network intelligence. This way, a new level of network flexibility is achieved, providing the means for on-the-fly re-configurability and optimization, which is crucial for real-time applications, going towards the implementation of new generation of services, such as Future Internet of Vehicles [27] in field of transportation.

In what follows, several projects will be presented, while the elements of collective cognitive computing can be identified within some of them.

DITAS Cloud Platform [28] allows developers to design data-intensive applications, deploy and execute them on heterogeneous devices in a mixed environment (Cloud/Edge) by exploiting the data and computation movement strategies. Most significant use cases of this framework come from healthcare domain [29], where large amount of patient monitoring data has to be processed timely, while maintaining the patient's privacy.

On the other side, a group of researchers from Australia proposed iFogSim [30], which is a simulation environment aiming the usage as an evaluation platform providing the capabilities related to the quantification of performance achieved as a result of adoption of resource management policies within Fog computing infrastructures relying on IoT devices in a repeatable manner. The impact of resource management techniques from several perspectives is taken into account: latency, network congestion, energy consumption, and cost.

MODAClouds [31] is a model-driven approach whose goal is to support the design of multi-Cloud applications with guaranteed QoS. It consists of contributions from several Horizon 2020 projects [32] and relies on a set of tool-supported domain- 
specific languages (DSLs), covering various aspects of Cloud computing [31]: service definition modelling, usage model, service orchestration, requirements modelling, data modelling and QoS modelling.

CAMEL (Cloud Application Modelling and Execution Language) [33] enables the management of self-adaptive cross-Cloud applications that autonomously adapt to changes in the environment, requirements, and usage. Its main focus is on the integration of heterogeneous domain-specific languages (DSLs) that cover different aspects of self-adaptive cross-cloud applications.

SMADA-Fog [34] is a semantic-enabled, modeldriven framework for modelling, simulation, deployment and adaptivity of services within Fog computing architectures to wide range of heterogeneous devices, from traditional servers and laptops, to IoT devices like Raspberry Pi. The smallest deployment unit is a task, which corresponds to a Docker container and is deployed to a server. The task can be either application service or network function. Moreover, the domain-specific modelling notation also considers the adaptive behavior of infrastructure and services in a particular context. This way, user is able to define the so-called adaptation strategy which consists of a set of actions taken when some pre-defined state is identified. For this purpose, when it comes to state identification (such as large number of service users predicted), the data analysis techniques performed against the monitoring data based on deep learning are leveraged. For example, when Quality of Service (QoS) value drop below given threshold is identified, the services can be scaled-up or redeployed making use of linear optimization model. The goal of the underlying optimization model is to provide the benefits to the both service providers and consumers, by maximizing the QoS, while minimizing the computing infrastructure maintenance costs (such as energy consumption cost). At the same time, the constraints related to data movement have to be satisfied - some of the tasks must be deployed on server residing within the Edge imposed by legal regulations. When it comes to networking, the containerized VNFs are adopted, while SDN rules aiming the QoS improvement are generated as result of runtime environment changer. SMADA-Fog was created as a side product of RAWFIE-SCOR [35] project, whose goal was the development of coordination mechanisms within RAWFIE [36] future internet platform for remote robotic experimentation.

RAWFIE-SCOR refers to a semantic technologybased framework aiming the extension of the existing robotic testbed capabilities, by introducing the modelling aspects related to device coordination [37]. Once the flow of coordination actions is defined and experiment executed, the central entity monitors and analyses the sensor data coming from drones and ground robots in order to detect the coordination points. Coordination points are events which trigger some scenario (a set of coordinated actions) as a response - such as fire in a building or object detection. This way, it is enabled to the robotics researchers to execute complex experiments including the coordinative behavior remotely, relying on future Internet services and Fog computing.

In both SMADA-Fog and RAWFIE-SCOR, a common adaptation model incorporating the elements of GCI was used. A similar adaptation concept can also identified in CAMEL (Cloud Application Modelling and Execution Language) [33]. The illustration of the generalized coordination model is given in Fig. 4.

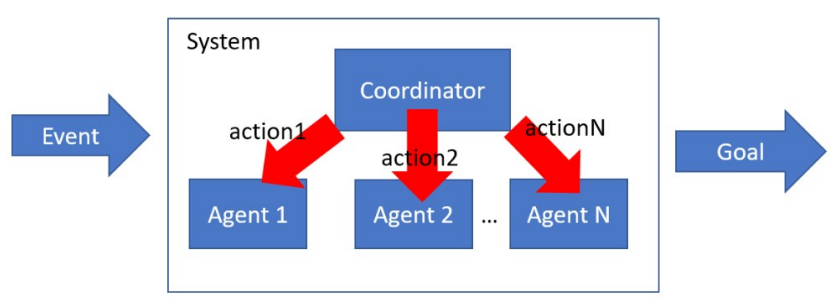

Figure 4. Common GCI adaptation model adopted in SMADA-Fog and RAWFIE-SCOR.

The response to the event which occurs within the environment is defined by adaption rule in form if(event) then goal. When event occurs, then centralized coordination of $n$ agents within the 
system is performed. Coordinator decomposes the goal in a set of actions, so each agent i $_{i}$ performs action $_{i}$ in order to contribute to the goal. The

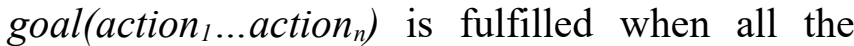
agents finish the action action $_{i}$ assigned to them by central coordination entity.

Finally, when it comes to adoption of Future Internet services within smart cities, in [38] and [39], modelling and simulation frameworks aiming the optimization of mobile network performance and energy efficiency (energy trading and autonomous vehicle charging) were presented. They rely on a set of domain-specific notations implemented within modelling tools and linear optimization for optimal resource planning, while blockchain is proposed for transactions (such as energy trading) [39]. In this notation, the main concepts are service infrastructure provider and service consumer. Service provider is represented as a set of network devices and servers able to provide some usage capacity, while consumers are represented as a side demanding some portion of its capacity, under given environment constraints. The complete simulation environments are accessible via web browser. Moreover, an extension of these tools in context of services tackling the current COVID-19 pandemic was presented in [40].

\section{Method}

The research question was addressed using the methods summarized in table 2 .

Table 1. Research question and method.

\begin{tabular}{|c|c|}
\hline Research Question & Method \\
\hline $\begin{array}{l}\text { 1. Is cognitive } \\
\text { computing or } \\
\text { collective cognitive } \\
\text { computing likely to } \\
\text { be a critical aspect } \\
\text { of different visions } \\
\text { of the future } \\
\text { Internet, even where, } \\
\text { or particularly where }\end{array}$ & $\begin{array}{l}\text { 1. An analysis is } \\
\text { performed to assess } \\
\text { whether and how } \\
\text { AGI and GCI might } \\
\text { be used to optimize } \\
\text { achievement of the } \\
\text { EU's vision for a } \\
\text { future Internet. } \\
\text { 2. An analysis is }\end{array}$ \\
\hline
\end{tabular}

\begin{tabular}{ll}
\hline these visions differ & performed to \\
wildly? & determine whether \\
& and if so how AGI \\
& and GCI might be \\
& used to optimize \\
& achievement of \\
& China's potentially \\
& very different vision \\
& for a future Internet. \\
\hline
\end{tabular}

5.1. Optimizing Outcomes for the European Union's Next Generation Internet Initiative

In the case of the EU's NGI initiative, the goals of the initiative might be best represented by the calls for proposals that were open at the time of writing of this paper, and that were related to Internet infrastructure rather than program administration:

Table 2. Goals of the EU's NGI Initiative

\begin{tabular}{ll}
\hline NGI Call for & Project \\
Proposal & \\
\hline FEDERATED & FED4FIRE+ \\
TESTBEDS FOR & \\
NGI & \\
INNOVATORS & \\
\hline BLOCKCHAIN & ONTOCHAIN \\
TECHNOLOGIES & \\
\hline DATA & NGI DAPSI \\
PORTABILITY & \\
AND SERVICES & \\
\hline TECHNOLOGIES & NGI ZERO \\
IMPROVING & DISCOVERY \\
SEARCH AND & \\
DISCOVERY & \\
\hline PRIVACY- & NGI ZERO PET \\
ENHANCING & \\
TECHNOLOGIES & \\
\hline SELF-SOVEREIGN & NGI ESSIF-LAB \\
IDENTITY & \\
\hline
\end{tabular}


Looking at the ONTOCHAIN, and NGI DAPSI projects as examples provides some insight into how cognitive and collective cognitive computing might be applied to maximize these goals. The ONTOCHAIN website [5] describes the ONTOCHAIN concept as "a new software ecosystem for trusted, traceable \& transparent ontological knowledge". The NGI DAPSI website [6] describes the NGI DAPSI concept as "interoperable solutions that enable data portability and tackle the following issues: service portability, data compatibility \& interoperability, security \& privacy, other topics". A service portability solution is described as one that can "empower users to share their data with any service provider and host that they trust". A data compatibility \& interoperability solution is described as one that can "facilitate switches between service providers". A security \& privacy solution is described as one that can "ensure the security \& privacy of consumers when their personal data are transferred from one provider to another". And the category "other topics" is reserved for any solution able to "solve relevant challenges in the data portability and services field".

The functionality required to implement a cognitive computing application, or to implement a collective cognitive computing application (a cognitive computing platform) have been described in the functional requirements for a proposed Peer to Peer Social Fabric (P2PSF). The $\mathrm{P} 2 \mathrm{PSF}$ is intended to act as the framework for any cognitive computing application, as the client to access any cognitive computing platform, and as the operating system enabling a GCI to orchestrate cooperation to execute processes in any cognitive computing platform. The functionality proposed for this platform includes application domains containing both the ONTOCHAIN and NGI DAPSI projects. Assuming AGI can be used to maximize individual user outcomes in any cognitive computing domain, and assuming GCI can be used to maximize collective user outcomes in any cognitive computing domain, this suggests that the proposed P2PSF has the functionality required to maximize achievement of the goals of the NGI program.
Cognitive computing and collective cognitive computing are therefore likely critical to all of the NGI challenges. From the functional modeling perspective any computing process can be represented as automating some sequence of activities in order to achieve some targeted outcomes. In order to maximize the complexity in which computing operations can be composed in order to in turn maximize those outcomes, and in order to remove the limits to the speed and scale at which components of a cognitive computing system can cooperate to increase these maximum outcomes, it is necessary in these functional models of cognitive computing and collective cognitive computing to decouple the properties of any activity from any instance of that activity's execution. For example, rather than an executing instance of a process being bound to a physical instance of a computing device, it must be bound to a virtual context so that the physical computing environment might be changed to another cooperating device. Rather than an executing instance of a process being bound to a user identity, it must be bound to a virtual identity so that execution can be transferred to another cooperating individual. These requirements arise because these functional models of cognitive and collective cognitive computing are based on the architecture of the human brain. Software processes from this perspective represent the automation of individual or collective reasoning processes. In a system of cognitive or collective cognitive computing computation then mimics the functionality through which the brain gains the capacity to scale its ability to navigate complexity, and by which it scales its ability to orchestrate cooperation between functional components in a massively decentralized way.

In order to have the capacity to operate in a massively decentralized way, in this functional model the brain must decouple the properties of reasoning processes from any instance of any reasoning process, so that reasoning cannot be forced to become centralized in a way that would prevent recruitment of the rest of the brain. For this reason, and because GCI involves orchestrating cooperation between individuals 
through different computing devices, the functional model of GCI provide far more comprehensive and self consistent requirements for properties such as decentralized identity, decentralized representation and storage of data, decentralized search and discovery, as well as privacy [7]. Because GCI based processes selfassemble into solutions that can be far more complex, with far more components, assembled using processes that can be sustained far larger than are possible for any individual or group without GCI, the requirements for these functional components of the NGI that are elaborated through GCI based processes in order to maximize collective outcomes, are likely not possible for any individual or group without GCI. With GCI the individualization of services to maximize collective outcomes follows a pattern [15]. The application of such a pattern to define a "cognitive blockchain" platform that might implement the "ONTOCHAIN" described by the NGI initiative has been explored elsewhere [8].

The takeaway is that this model suggests that the functionality required to maximize the ability to implement the future Internet is the functionality required for both adaptive problem-solving and for massively increasing that problem-solving ability, such as through facilitating massively decentralized cooperation. Since GCI might be required to achieve AGI [14], GCI provides what is believed to be the only comprehensive model for both. By extension, this implies that without GCI hidden bugs (or intentional features) in any implementation must prevent optimal success in the stated goals of the future Internet from being reliably achievable [13].

\subsection{Optimizing Outcomes for China's Next Generation Internet Initiative}

Given China's strategy of defining optimal use of the Internet and collectively enforcing that optimal use, perhaps exemplified by the country's strategy of segregating the Internet of the country from the external world using a national firewall, China's goals for the Internet of the future might focus more around the ability to individually monitor and control each node on the Internet in a centralized way. As always, cognitive computing can still maximize outcomes, but in this case it's important to recognize that the outcomes being optimized are those chosen by central government.

At first glance it might appear that in every case in which maximizing outcomes for the end user conflicts with maximizing collective outcomes for the Internet owner, a cognitive Internet platform must be barred. Of course, this would have significant side effects, since GCI provides the capacity for a potentially exponential increase in impact on any targeted collective outcomes in general. As a consequence, any country that fails to adopt GCI must be at a significant disadvantage in health care, education, economic development, and every other aspect of the operation of a sovereign country.

A deeper look reveals that rather than needing to bar the cognitive Internet, the opposite might be true. China's vision for the future Internet might be maximized by the cognitive Internet, since GCI is an adaptive problem-solving system that can decentralize where optimal according to the values of the individuals in any implementing country itself, and can centralize where optimal according to the values of that country [15]. GCI has no political agenda. GCI is maximally aligned with the goals of any country to maximizing collective well-being according to what that country deems serves its well-being, whether the national philosophy is that maximizing collective well-being can only be achieved through ensuring individual rights and freedoms, or whether the national philosophy is that maximizing collective well-being can only be achieved through enforcing responsibility to what the government believes is in the interest of the common good. GCI merely ensures self-consistency across all initiatives so they might reliably converge on that collective well-being.

An article in wikipedia provides more detail regarding this speculation regarding China's potential goals for the future Internet not just for China, but globally. According to a brochure 
entitled "CNGI-CERNET2/61X", the key tasks of the China Next Generation Internet (CNGI) were:

- Construction of China's next generation Internet backbones

- Development of key network technology and major applications for the next generation Internet

- Promotion of industrialization and application development of next generation Internet equipment and software

China's goal of spreading this centrally controlled vision worldwide might be reflected in the statement: "Participation in international organizations, and playing an important role in standards setting IPv6 was selected as key technology. The United States has almost one third of the theoretical maximum IPv4 addresses (about 4.3 billion),[1] while China has more highspeed Internet users than IP addresses and the largest Internet user base of any country.[2] With the implementation of IPv6, China planned to avoid imminent problems of IPv4 address exhaustion".

The article goes on to state that "everything from security cameras, taxis, to the Olympic events cameras are networked by IPv6; the events are streamed live over the Internet while networked cars are able to monitor traffic conditions readily". This appears again to be a centralized vision of the Internet in which networked cars are able to share information in a way that enables a centralized application to "monitor traffic conditions". In the absence of GCI, from the perspective of functional modeling the vision of global cooperation translates to a vision of centralized global control. With GCI cooperation might be decentralized, but this model predicts that far more powerful cooperation can be targeted at any goal. With GCI individuals can self-assemble into networks of cooperation that are far more complex and with cooperation that is able to scale outcomes to a far greater degree, than possible for any individual or groups of individual without GCI to have the capacity to design.

\section{Findings}

A brief analysis shows the various forms of modelling such as Domain Specific Modelling have been successfully applied to the purpose of implementing the future Internet. From the functional modelling perspective, cognitive computing applications leverage AGI to optimize user outcomes, and cognitive computing platforms leverage GCI to optimize collective user outcomes. Since cognitive computing platforms leverage GCI to enable intelligent agents based on AGI to self-assemble into solutions, GCI appears to create the possibility of future Internet technology that self-assembles in different ways according to the users, so that it simultaneously might maximize outcomes in implementing potentially conflicting visions of that future Internet.

Table 3. Research Findings.

\begin{tabular}{ll}
\hline Research Question & Findings \\
\hline 1. Is cognitive & The answer \\
computing or & appears to be yes \\
collective cognitive & pending \\
computing likely to be & confirmation by an \\
a critical aspect of & actual \\
different visions of the & implementation. \\
future Internet, even & \\
where, or particularly & \\
where these visions & \\
differ wildly? & \\
\hline
\end{tabular}

\section{Research Limitations}

Neither GCI nor a functional model of the Internet have not yet been completely implemented. As a result, this analysis has been conducted at a relatively abstract level. Actual implementation may reveal missing details.

\section{Practical Implications}

Creating in effect 7.5 billion Internet's, one for each person in the world, would seem to require an unfeasible amount of computing and storage 
resources. However, the potential to define such configurations so they are optimally shared remains to be explored. And in addition, the potential of GCI to combine such services into networks of cooperation [41] that create sufficient value to sustain those resources, is a direction that might hold promise.

\section{Conclusions}

General Collective Intelligence is a comprehensive vision that might be too large to justify within the budget of any single project. However, GCI also creates the potential to significantly increase impact for a wide range of projects, including those related to the future Internet. Through spreading the implementation of GCI over multiple projects, the budget per project might be reliably reduced to a level that is feasible for each. As explored in this paper, there is significant motivation to explore the possibility of doing so. Without GCI, efforts to implement the future Internet must contain hidden bugs (or intentional features) that are predicted to prevent the stated goals of the future Internet from being reliably achievable for any country regardless of their vision of that future Internet.

Since GCI has the potential to exponentially increase impact on any outcomes, building a GCI based future Internet that can exponentially increase China's power to ensure harmonious use of the Internet by the entire population, and building an Internet for the West that is exponentially more secure and free from censorship, are completely consistent.

The exponential increase in power to achieve any outcome that might be possible through a GCI based future Internet is power that can be directed at any outcome desired by the group. Just like big data might have the capacity to customize marketing services for every single individual in the 330 million population of the US, a GCI based future Internet could have the capacity to customize the Internet for every individual as well. For individuals who prioritize individual rights and freedoms, that functionality will enable them to self-assemble into an Internet in which censorship and surveillance is impossible. For individuals who prioritize group values, that functionality will enable them to self-assemble into an Internet in which content offensive or contrary to those values is completely eliminated. As explored in more detail elsewhere, humans have irreconcilable biases towards understanding truth in terms of individual rights and freedoms, or in terms of consistency with group values. In the US these biases might correspond to alignment of political views with what in the US is considered the political left or the political right [16]. Between China and the US these biases might correspond to correspond to alignment with the political system of the CCP or with the political system of the US. Because these biases are irreconcilable, no argument as to which is wrong or right can be successful at convincing the other side.

Rather than China and the US having conflicting goals in implementing the future Internet, a GCI based future Internet designed by China will also fit the needs of the West. By taking this stance, it then becomes possible to launch large-scale future Internet projects that leverage public funding both in China, as well as in the West. In essence, the future Internet in this vision can use exactly the same technologies but self-assemble in ways that look entirely different depending on what one's own biases suggest the optimal Internet should look like. At the same time, the GCI will identify the Internet features that other users have leveraged to gain better outcomes than one might have achieved, so that even though one's own biases might initially cause groups to selfassemble in ways that restrict the rights and freedoms of others, or initially cause groups to self-assemble in ways that remove the ability of the group to be protected from that which is contrary to the collective interests, with the assistance of the GCI the future Internet might reliably converge on a configuration that more optimally achieves the goals each community itself has identified. 
Acknowledgements.

Thanks to Julian Talev for countless discussions on the topic.

\section{References}

1 Website: "Overview of Country IP Usage", BGPExperts.com, May 7, 2007

2 Website: "China Surpasses U.S. In Internet Use", Forbes.com, March 4, 2006

3 Website: Beijing2008.cn. "Beijing 2008 Archived 2009-02-04 at the Wayback Machine." Beijing2008.cn leaps to next generation Net. Retrieved on 2008-11-09.

4 Website: China Next Generation Internet, Wikipedia

5 Website: ONTOCHAIN, retrieved from https://ontochain.ngi.eu/, accessed on Nov 11,2020

6 Website: NGI DAPSI, retrieved from https://dapsi.ngi.eu/, accessed on Nov 11, 2020

7 Pre-print: Williams, A. E. (n.d.). The Peer to Peer Social Fabric as a Platform for General Collective Intelligence. Retrieved from osf.io/preprints/africarxiv/qbxfr

8 Pre-print: Andy E. Williams, Roberto Moro visconti, Andy E. Williams, The Application of Artificial General Intelligence to the Cognitive Blockchain and the Internet of Value, DOI: 10.13140/RG.2.2.15004.31363

9 Pre-print: Williams, A. E. (2020, May 15). Use of Human-Centric Functional Modeling to Maximize Convergence in Integrative Research. https://doi.org/10.31730/osf.io/jv6h8

10 Book Chapter: Williams A.E. (2020) A Model for Artificial General Intelligence. In: Goertzel B., Panov A., Potapov A., Yampolskiy R. (eds) Artificial General Intelligence. AGI 2020. Lecture Notes in Computer Science, vol 12177. Springer, Cham. https://doi.org/10.1007/978-3-03052152-3_38

11 Conference: The Relationship Between Collective Intelligence and One Model of General Collective Intelligence, Andy E.
Williams, Computational Collective Intelligence, 11th International Conference, ICCCI 2019, Hendaye, France, September 4-6, 2019, Proceedings, Part II, Pages 589600

12 Pre-print: Williams, A. E. (2020, November 11). General Collective Intelligence and the Transition to Collective Super-Intelligence. https://doi.org/10.31730/ osf.io/tacyq, under review (2020)

13 Pre-print: Williams, A. E. (2020, April 16). General Collective Intelligence and the Constraints to Group Decision-Making. https://doi.org/10.31234/osf.io/6gten

14 Pre-print: Williams, A. E. (2020, August 17). Does Creating an Artificial General Intelligence Require General Collective Intelligence in Order to be Reliably Achievable?.

https://doi.org/10.31730/osf.io/3q72c

15 Pre-print: Williams, A. E. (2020, December 16). Individualization of Products and Services with Artificial General Intelligence and General Collective Intelligence. https://doi.org/10.31730/osf.io/ gd5mt

16 Pre-print: Williams, A. E. (2020, November 17). Bridging the Left - Right Political Divide with Artificial Intelligence and Collective Intelligence. https://doi.org/10.31730/osf.io/y65x4

17 Journal article: Krafft, P.M. (2019), A Simple Computational Theory of General Collective Intelligence. Top Cogn Sci, 11: 374-392.

https://doi.org/10.1111/tops.12341.

18 Journal article: Woolley, Anita Williams; Chabris, Christopher F.; Pentland, Alex; Hashmi, Nada; Malone, Thomas W. (29 October 2010). "Evidence for a Collective Intelligence Factor in the Performance of Human Groups". Science. 330 (6004): 686688. Bibcode:2010Sci...330..686W. doi:10.1126/science.1193147. PMID 20929725. S2CID 74579.

19 Book: Malone, T. W. (2018). Superminds: The surprising power of people and computers thinking together. 
20 Website: Horizon 2020 projects [online]. Available on: https://ec.europa.eu/programmes/horizon20 20/en/h2020-sections-projects , last accessed: 18/12/2020.

21 Book Chapter: D. Bermbach et al., "A Research Perspective on Fog Computing", In: Service-Oriented Computing - ICSOC 2017 Workshops, Lecture Notes in Computer Science, vol 10797. Springer, Cham, pp. 198-210, 2018. https://doi.org/10.1007/978-3-319-91764$1 \_16$

22 Website: General Data Protection Regulation [online]. Available on: https://gdpr-info.eu/, last accessed: 18/12/2020.

23 Journal article: M. Aazam, S. Zeadally, K. A. Harras, "Deploying Fog Computing in Industrial Internet of Things and Industry 4.0", IEEE Transactions on Industrial Informatics ( Volume: 14, Issue: 10, Oct. 2018), pp. 4674 - 4682, 2018. https://doi.org/10.1109/TII.2018.2855198

24 Conference: C. M. Kanaka Sri Shalini, Y. M. Roopa, J. S. Devi, "Fog Computing for Smart Cities", 2019 International Conference on Communication and Electronics Systems (ICCES), pp. 911-916. doi:10.1109/icces45898.2019.9002050

25 Conference: D. N. Palanichamy, K. I. Wong, "Fog Computing for Smart Grid Development and Implementation", 2019 IEEE International Conference on Intelligent Techniques in Control, Optimization and Signal Processing (INCOS), pp. 1-6. doi:10.1109/incos45849.2019.8951412

26 Book Chapter: D. Kim et al., "Integrated SDN and Non-SDN Network Management Approaches for Future Internet Environment", In: Multimedia and Ubiquitous Engineering. Lecture Notes in Electrical Engineering, vol 240. Springer, Dordrecht, pp. 529-536, 2013. https://doi.org/10.1007/978-94-007-6738664

27 Journal: C. A. Kerrache, M. Amadeo, S. H. Ahmed, C. Liang, "Future Internet of
Vehicles", Transactions on Emerging Telecommunications Technologies 2020; e3975, pp. 1-2, 2020. https://doi.org/10.1002/ett.3975

28 Website: DITAS Cloud Platform [online]. Available on: https://www.ditas-project.eu/, last accessed 18/12/2020.

29 Journal article: C. Cappiello et al., "Improving Health Monitoring With Adaptive Data Movement in Fog Computing", Front. Robot. AI, 15 September 2020, pp. 1-19. https://doi.org/10.3389/frobt.2020.00096

30 Journal article: H. Gupta et al., "iFogSim: A toolkit for modeling and simulation of resource management techniques in the Internet of Things, Edge and Fog computing environments". Software: Practice and Experience, 47(9), 1275-1296, 2017. https://doi.org/10.1002/spe.2509

31 Book Chapter: N. Ferry, M. Almeida, A. Solberg, "The MODAClouds Model-Driven Development", In: Model-Driven Development and Operation of Multi-Cloud Applications. SpringerBriefs in Applied Sciences and Technology. Springer, Cham, pp. 23-33, 2017. https://doi.org/10.1007/978-3-319-460314_3

32 Website: MultiCloud DevOps Toolbox [online]. Available on: http://multiclouddevops.com/ , last accessed: 18/12/2020.

33 Website: A. Rossini et al., "The Cloud Application Modelling and Execution Language (CAMEL)", OPen Access Repository of Ulm University (OPARU), pp. $1-39$, 2017. https://doi.org/10.18725/OPARU-4339

34 Journal article: N. Petrovic, M. Tosic, "SMADA-Fog: Semantic model driven approach to deployment and adaptivity in Fog Computing", Simulation Modelling Practice and

Theory, 102033, pp. 1-25, 2019. https://doi.org/10.1016/j.simpat.2019.10203 3

35 Website: Semantic Coordination for RAWFIE [online]. Available on: 
http://infosys1.elfak.ni.ac.rs/scor/, last accessed: 18/12/2020.

36 Website: Road-, Air-, and Water- based Future Internet Experimentation [online]. Available on: http://rawfie.eu/ , last accessed: 18/12/2020.

37 Journal article: V. Nejkovic, N. Petrovic, M. Tosic, N. Milosevic, "Semantic approach to RIoT autonomous robots mission coordination", Robotics and Autonomous Systems 126:103438, pp. 119 , 2020. https://doi.org/10.1016/j.robot.2020.103438

38 Journal article : N. Petrović, Đ. Kocić, "Data-driven Framework for EnergyEfficient Smart Cities", Serbian Journal of Electrical Engineering, Vol. 17, No. 1, Feb. 2020, pp. 41-63, 2020. https://doi.org/10.2298/SJEE2001041P

39 Conference: N. Petrović, S. Koničanin, D. Milić, S. Suljović, S. Panić, "GPU-enabled Framework for Modelling, Simulation and Planning of Mobile Networks in Smart Cities", ZINC 2020, pp. 280-285, 2020. https://doi.org/10.1109/ZINC50678.2020.91 61773

40 Conference: N. Petrović, "Simulation Environment for Optimal Resource Planning During COVID-19 Crisis", ICEST 2020, pp. 23-26, 2020. https://doi.org/10.1109/ICEST49890.2020.9 232908

41 Pre-print: Williams, A. E. (2020, December 16). Discovering and Implementing Self-Sustaining Networks of Cooperation with General Collective Intelligence. https://oi.org/10.31730/osf.io/ safxk 